\title{
ARTICLE
}

\section{Measurement of double differential neutron yields from thick carbon target irradiated by 5-MeV and 9-MeV deuterons}

\author{
Yuta Tajiri $^{\mathrm{a}}$, Yukinobu Watanabe ${ }^{\mathrm{a}^{*}}$, Nobuhiro Shigyo ${ }^{\mathrm{b}}$, Keiichi Hirabayashi ${ }^{\mathrm{b}}$, Tomoya Nishizawa ${ }^{\mathrm{b}}$ and Kenshi Sagara ${ }^{\mathrm{c}}$ \\ ${ }^{a}$ Department of Advanced Energy Engineering Science, Kyushu University, 6-1 Kasuga-koen, Kasuga, Fukuoka, 816-8580, Japan; \\ ${ }^{\mathrm{b}}$ Department of Applied Quantum Physics and Nuclear Engineering, Kyushu University, 744 Motooka, Nishi-ku, Fukuoka, \\ 819-0395, Japan; 'Department of Physics, Kyushu University, 6-10-1 Hakozaki, Higashi-ku, Fukuoka, 812-8581, Japan
}

\begin{abstract}
Double differential neutron yields from carbon target irradiated by $5 \mathrm{MeV}$ and $9 \mathrm{MeV}$ deuterons were measured at the Kyushu University Tandem Accelerator Laboratory. A carbon target was thick enough to stop the incident deuterons in the target. An NE213 liquid organic scintillator was employed to detect neutrons emitted from the target and placed at several directions from $0^{\circ}$ to $140^{\circ}$. To estimate the contribution of scattered neutrons from the floor and walls, neutron yields were measured with an iron shadow bar located in front of the scintillator. The measured energy spectrum was derived by an unfolding method with FORIST and MAXED codes using the response functions of the NE213 scintillator calculated with the SCINFUL-QMD code. The incident energy dependence of total thick target neutron yields for carbon was investigated over the energy range from 5 to $50 \mathrm{MeV}$ and the empirical formula was obtained. The measured neutron energy spectra were also compared with the calculations by PHITS code.
\end{abstract}

Keywords: measurement; deuteron; neutron; thick target yield; unfolding; carbon; PHITS

\section{Introduction}

In recent year, attention has been focused on deuteron accelerator based neutron sources in various applications such as boron neutron capture therapy (BNCT) and medical radioisotope production [1]. In these applications, detailed information on neutrons generated in the target is required to determine accurately the neutron source term necessary for shielding design and radiation dose estimation. Carbon is considered as one of the candidates for target materials. However, experimental data of double differential thick target neutron yields (DDTTNYs) do not exist below $10 \mathrm{MeV}$.

The purpose of this study is to measure the DDTTNYs for both $5 \mathrm{MeV}$ and $9 \mathrm{MeV}$ deuteron incidences on the carbon target. The experimental results are compared with the calculation made by the PHITS code. Incident energy independence on neutron thick target yields is investigated using the results of the present measurement and others.

\section{Experiment}

\subsection{Experimental setup}

Details of the experimental set up have been reported in Ref. [2]. The experiment was performed at the $1 \mathrm{st}$ target room in the Kyushu University Tandem

\footnotetext{
* Corresponding author.Email: watanabe@aees.kyushu-u.ac.jp
}

Accelerator Laboratory. The experimental setup is illustrated in Figure 1.

The 5 and $9 \mathrm{MeV}$ deuteron beam from the tandem accelerator was delivered to a compact vacuum target chamber in the target room. The chamber was insulated from other experimental apparatus and the ground of the experimental room in order to acquire the deuteron beam current.

The target chamber $260 \mathrm{~mm}$ in diameter equipped a target frame which enabled to mount up to four target foils at the center of the chamber. A 2-cm height aperture was set at the beam line height of the chamber in order to reduce neutron scattering at the stainless wall of the chamber. The aperture was covered with a 125 $\mu \mathrm{m}$ thick Mylar film to keep a vacuum inside the chamber.

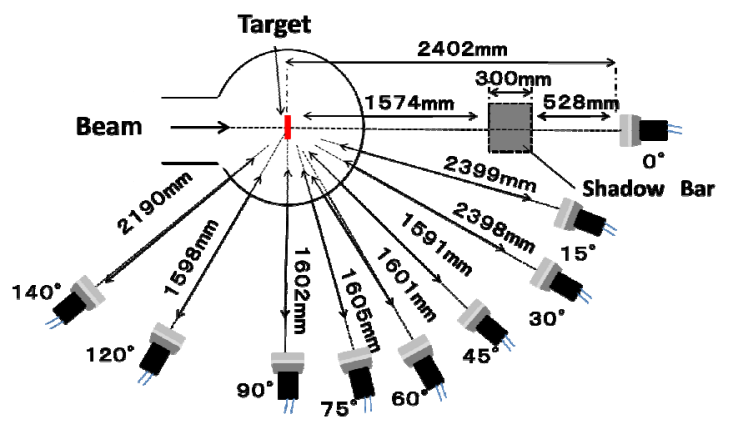

Figure 1. Experimental set up 
The target thickness was chosen so that incident deuterons stop completely in the target based on the range calculation with the SRIM code [3]: $0.4 \mathrm{~mm}$ for 5 $\mathrm{MeV}$ and $0.5 \mathrm{~mm}$ for $9 \mathrm{MeV}$, respectively.

An NE213 liquid organic scintillator $50.4 \mathrm{~mm}$ thick and $50.4 \mathrm{~mm}$ in diameter was adopted as a neutron detector. The detector was placed in the distance from 1.6 to $2.4 \mathrm{~m}$ from the target. Neutron yields from the target were measured at six angles of $0^{\circ}, 30^{\circ}, 60^{\circ}, 90^{\circ}$, $120^{\circ}$ and $140^{\circ}$ for $5 \mathrm{MeV}$, and nine angles of $0^{\circ}, 15^{\circ}$, $30^{\circ}, 45^{\circ}, 75^{\circ}, 90^{\circ}, 120^{\circ}$ and $140^{\circ}$ for $9 \mathrm{MeV}$.

In order to estimate the contribution of neutrons from the floor and walls in the experimental room, a measurement with an iron shadow bar $150 \mathrm{~mm} \times 150$ $\mathrm{mm} \times 300 \mathrm{~mm}$ thick placed between the target and the neutron detector was performed for each direction as a background run.

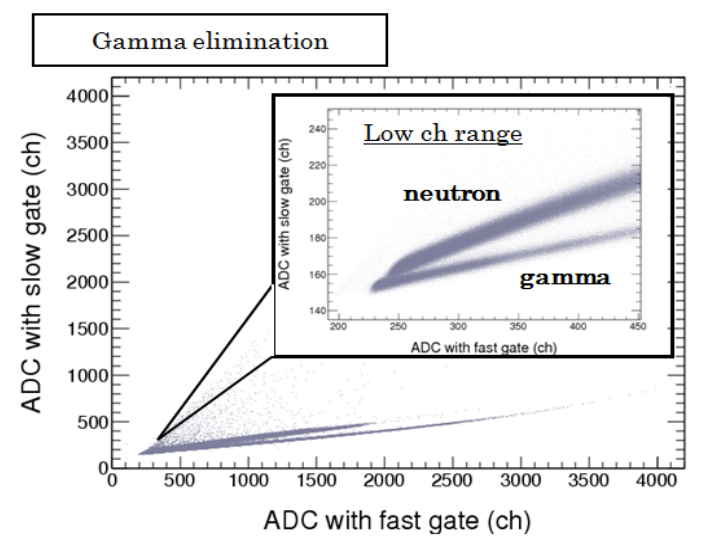

Figure 2. Two dimensional plot of neutron and $\gamma$ ray discrimination using the two gate integration method.

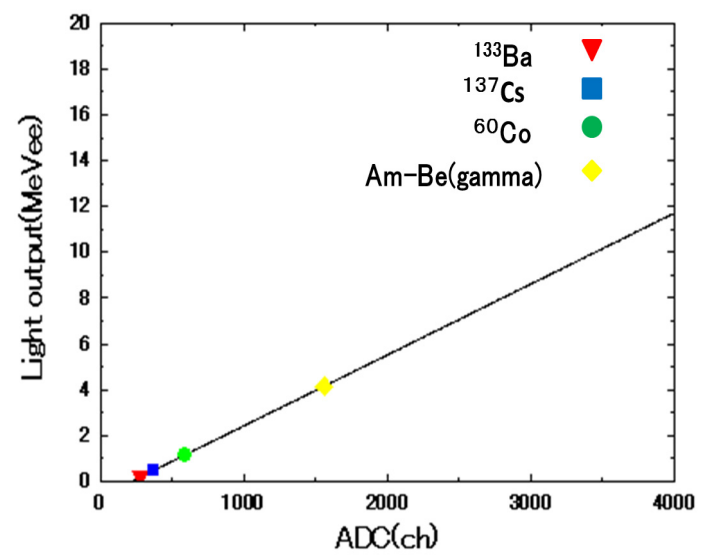

Figure 3. Relationship between integrated charge and light output.

\subsection{Data Analysis}

First, $\gamma$ ray events were separated from light output spectra using the two gate integration method because the NE213 scintillator is sensitive to $\gamma$ rays in addition to neutrons. Figure 2 shows a two dimensional plot of neutron and $\gamma$ ray discrimination. One can see that neutron events are separated well from $\gamma$ ray ones in low light output region in the figure.

Second, the light output spectra of neutron events were converted to the amount in units of electron equivalent using $\gamma$ rays from ${ }^{133} \mathrm{Ba}(\mathrm{E}=0.36 \mathrm{MeV}),{ }^{137} \mathrm{Cs}$ $(\mathrm{E}=0.66 \mathrm{MeV}),{ }^{60} \mathrm{Co}(\mathrm{E}=1.17$ and $1.33 \mathrm{MeV})$, and $\mathrm{Am}-\mathrm{Be}(\mathrm{E}=4.44 \mathrm{MeV})$ standard $\gamma$ ray sources. The calibration curve was given by fitting the ADC channels corresponding to the Compton edges for these $\gamma$ rays. The relationship between the charge recorded into an ADC and the light output is shown in Figure 3.

In the present experiment, the time-of-flight method was not applied because a pulsed deuteron beam was not available at the accelerator facility. Therefore, the neutron energy spectra were obtained from an unfolding method using the response functions of the NE213 scintillator. The response functions were calculated by the SCINFUL-QMD code [4]. Figure 4 shows the calculated response functions. The unfolding of light output spectra were performed by the FORIST code [5] and the MAXED code [6]. These codes are based on the method of least squares and maximum entropy method, respectively.

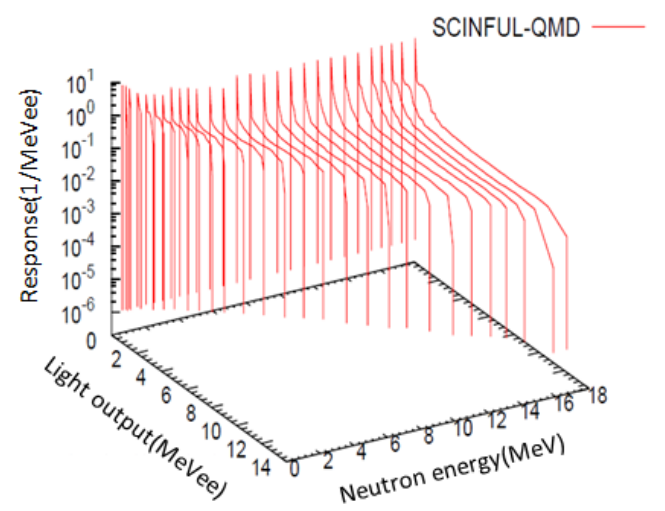

Figure 4. Response functions of NE213 scintillator calculated using SCINFUL-QMD code.



Figure 5. Measured double differential thick target neutron yields from carbon bombarded by $9 \mathrm{MeV}$ deuteron. 




Figure 6. Measured double differential thick target neutron yields from carbon bombarded by $5 \mathrm{MeV}$ deuteron.

\section{Results and discussion}

Experimental double differential thick target neutron yields (DDTTNYs) obtained using the FORIST code are shown in Figure 5 for $9 \mathrm{MeV}$ and Figure 6 for $5 \mathrm{MeV}$, respectively. The error bars include both the statistical error and the uncertainty caused by FORIST unfolding. The observed lowest neutron energy is approximately 1 $\mathrm{MeV}$. Below $2 \mathrm{MeV}$, the experimental errors tend to increase. At forward angles, two humps are observed around the incident energy and half the incident energy. Figure 7 shows a comparison of the DDTTNYs obtained using two codes based on different unfolding methods, FORIST and MAXED. It is found that both the results are in good agreement in both shape and magnitude. Therefore, the results with the FORIST code were used in the following analyses.

Energy-integrated thick target neutron yields at $0^{\circ}$ are shown in Figure 8 along with other experimental data [1, 7-10]. In the energy integration, the experimental yields below $1 \mathrm{MeV}$ corresponding to the lowest measured energy were supplemented by linear extrapolation with the data at $1 \mathrm{MeV}$. The empirical expression shown in the figure was obtained by a least-square fitting of all these experimental data.

Angular distributions of the measured neutron yields at $5 \mathrm{MeV}$ and $9 \mathrm{MeV}$ are plotted in Figure 9. Strongly forward-peaked distributions are observed clearly. The results were fitted well using the empirical formula proposed by Kalbach [11] as shown in the figure.

An experimental total neutron yield per incident deuteron was extracted by angle integration using linear extrapolation at angles larger than $140^{\circ}$. In Figure 10, both the results of 5 and $9 \mathrm{MeV}$ are plotted along with other experimental data [12-14]. The present measurement at $5 \mathrm{MeV}$ coincides with the past data [12] at the same energy, resulting in validation of our measurement.

In addition, the experimental data are compared with two PHITS calculations using the total cross sections calculated by the Shen formula [15] and by the NASA formula [16] in Figure 11 for $9 \mathrm{MeV}$ and $0^{\circ}$. The JQMD model was adopted as an option of nuclear reaction models. The use of the NASA formula underestimates the measured data remarkably, whereas the calculation with the Shen formula improves the agreement with the measurement although there is still underestimation.



Figure 7. Comparison of double differential thick target neutron yields obtained using the FORIST code and the MAXED code for $9 \mathrm{MeV}$ and $0^{\circ}$.

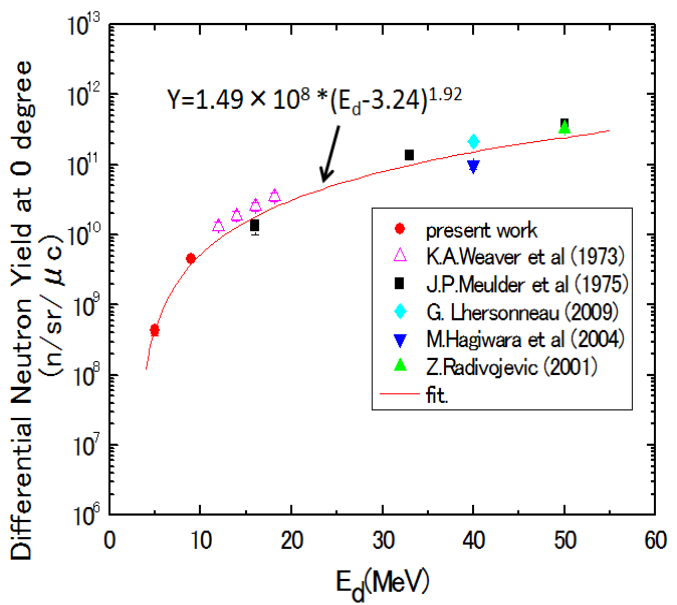

Figure 8. Energy-integrated thick target neutron yield at $0^{\circ}$ as a function of incident deuteron energy.

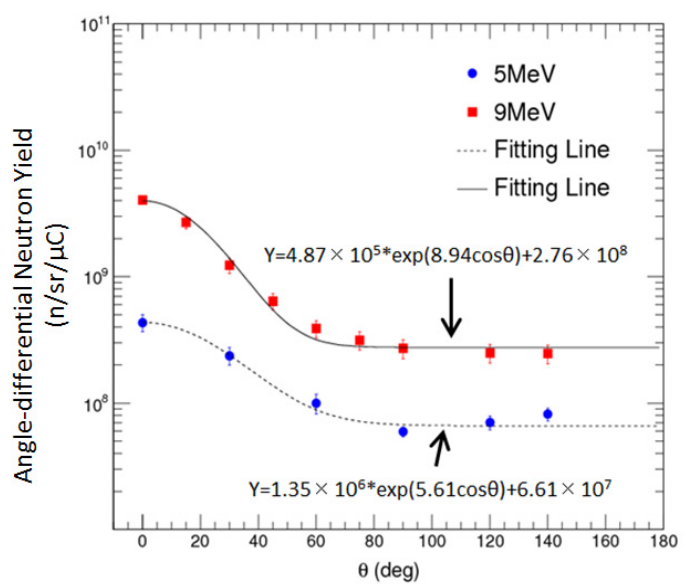

Figure 9. Angle-differential neutron yields for $5 \mathrm{MeV}$ and 9 $\mathrm{MeV}$ deuterons. 


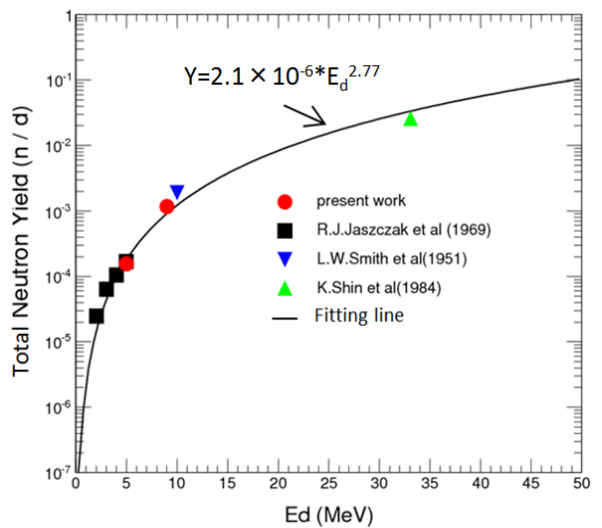

Figure 10. Total thick target neutron yield per incident deuteron for carbon as a function of incident deuteron energy.



Figure 11. Comparison of experimental double differential thick target neutron yields with the PHITS calculations.

\section{Summary}

The double differential neutron yields from $5 \mathrm{MeV}$ and $9 \mathrm{MeV}$ deuteron incidences on thick carbon target were measured using an NE213 liquid organic scintillator for reliable radiation dose evaluation at the high-power deuteron accelerator facility. The light output spectra were unfolded by the FORIST code in order to obtain the neutron energy spectra. By inclusion of other experimental data above $9 \mathrm{MeV}$, the empirical formulas were derived for prediction of differential neutron yields at $0^{\circ}$ and total neutron yield per incident deuteron over the energy range up to $50 \mathrm{MeV}$. It was found that the PHITS calculation with the Shen formula for total reaction cross sections reproduces the experimental data relatively better than that with the NASA formula.

\section{References}

[1] G.Lhersonneau, T. Malkiewicz, K. Kolos, M. Fadil, H. Kettunen, M.G. Saint-Laurent, A. Pichard, W.H. Trzaska, G. Tyurin and L. Cousin, Neutron yields from carbon, light- and heavy-water thick targets irradiated by $40 \mathrm{MeV}$ deuterons, Nucl. Instrum. and Meth. A 603 (2009), pp. 228-235.

[2] N. Shigyo, K. Hidaka, K. Hirabayashi, Y. Nakamura, D. Moriguchi, M. Kumabe, H. Hirano, S. Hirayama, Y. Naitou, C. Motooka, C. Lan, T.
Watanabe, Y. Watanabe, K. Sagara, S. Maebara, H. Sakaki and H. Takahashi, Measurement of Deuteron Induced Thick Target Neutron Yields at $9 \mathrm{MeV}$, Proc. International Conference on Nuclear Data for Science and Technology (ND2010), Jeju Korea, Apri. 26-30, 2010, (2011), pp. 1725-1728.

[3] J.F. Ziegler, J.P. Biersack and U. Linmark, The Stopping and Range of Ions in Solids, Pergamon Press, New York, (2009).

[4] D. Satoh, T. Sato, N. Shigyo and K. Ishibashi, SCINFUL-QMD: Monte Carlo based computer code to calculate response function and detection efficiency of liquid organic scintillator for neutron energies up to $3 \mathrm{GeV}$, JAEA-Data/Code 2006-023, Japan Atomic Energy Research Institute, (2006).

[5] R. H. Johnson, D.T. Iegersoll, B.W. Wehrin and J.J Dorning, NE-213 Neutron Spectrometry System for Measurement from 1.0 to $20 \mathrm{MeV}, \mathrm{Nucl}$. Instr. Meth. 145 (1977), pp. 337-346.

[6] M. Reginatto, B. Wiegel and A. Zimbal, UMG-Code Package, available from the Nuclear Energy Agency (NEA) Data Bank, http://www.nea.fr.

[7] K.A. Weaver, J.D. Anderson, H.H. Barschall and J.D. Davis, Neutron spectra from deuteron bombardment of d, Li, Be, and C, Nucl. Sci. Eng. 52 (1973), pp.35-45.

[8] J.P. Meulders, P. Leleux, P.C. Macq and C. Pirart, Fast neutron yields and spectra from targets of varying atomic number bombarded with deuterons from 16 to $50 \mathrm{MeV}$ (for radiobiology and radiotherapy), Phys. Med. Biol. 20 (1975), pp.235-243.

[9] M. Hagiwara, T Itoga, M Baba, M.S Uddin, N Hirabayashi, $\mathrm{T}$ Oishi and $\mathrm{T}$ Yamauchi, Experimental studies on the neutron emission spectrum and activation cross-section for $40 \mathrm{MeV}$ deuterons in IFMIF accelerator structural elements, J. Nucl. Materials 329-333 (2004), pp. 218-222.

[10]Z. Radivojevic, A. Honkanen, J. Äystö, V. Lyapin, V. Rubchenya, W.H. Trzaska, D. Vakhtin and G. Walter, Neutron yields from thick ${ }^{12} \mathrm{C}$ and ${ }^{9} \mathrm{Be}$ targets irradiated by $50 \mathrm{MeV}$ and $65 \mathrm{MeV}$ deuterons, Nucl. Instrum. and Meth. in Phys. B 183 (2001), pp.212-220.

[11]C. Kalbach, Systematic of continuum angular distributions: Extensions to higher energies, Phys.Rev.C 37 (1988), pp. 2350-2369.

[12]R.J. Jaszczak, R.L. Macklin and J.H. Gibbons, ${ }^{12} \mathrm{C}(\mathrm{d}, \mathrm{n}){ }^{13} \mathrm{~N}$ Total cross section from 1.2 to 4.5 MeV, Phys. Rev. 181 (1969), pp. 1428-1430.

[13]L.W. Smith and G. Kruger, Thick target yields from the $(\mathrm{d}, \mathrm{n})$ reaction at $10 \mathrm{MeV}$, Phys. Rev. 83 (1951), pp. 1137-1140.

[14]K. Shin, K. Hibi and M. Fujii, Neutron and photon production from thick targets bombarded by $30-\mathrm{MeV}$ p, 33-MeV d, 65-MeV ${ }^{3} \mathrm{He}, 65-\mathrm{MeV} \alpha$ ions: Experiment and comparison with cascade Monte Carlo calculations, Phys. Rev. C 29 (1984), 
pp. 1307-1316.

[15]R.K. Tripathi, F.A. Cucinotta and J.W. Wilson, Accurate universal parameterization of absorption cross sections III-light systems, Nucl. Instr. and Meth. B 155 (1999), pp. 349-356.
[16]W.Q. Shen, B. Wang, J. Feng, W.L. Zhan, Y.T. Zhu and E.P. Feng, Total reaction cross section for heavy-ion collisions and its relation to the neutron excess degree of freedom, Nucl. Phys. A 491 (1989), pp. 130-146. 